\title{
Ljubljana nomograms for predicting the likelihood of non-sentinel lymph node metastases in breast cancer patients with a positive sentinel lymph node
}

\author{
Andraž Perhavec $\cdot$ Maja Pohar Perme $\cdot$ \\ Marko Hočevar · Nikola Besić · Janez Žgajnar
}

Received: 5 August 2009/Accepted: 16 September 2009/Published online: 29 September 2009

(C) Springer Science+Business Media, LLC. 2009

\begin{abstract}
Several tools for predicting the likelihood of non-sentinel lymph node (non-SLN) involvement in SLNpositive breast cancer patients have been created so far. The aim of our study was to create and validate different nomograms for predicting the likelihood of non-SLN involvement that would be applicable in different institutions and that would also include the results of the preoperative US examination of the axilla. From January 2000 to January 2009, 534 breast cancer patients underwent axillary lymph node dissection (ALND) due to metastatic SLN at our institution. Using logistic regression results three nomograms differing in the inclusion of the results of intraoperative examination of SLN were created. The nomograms were validated using bootstrap methods. In all three nomograms, US examination of the axilla was a powerful independent variable. Other variables included (different in different nomograms) were tumor size, lymphovascular invasion, metastasis size in SLN, number of negative and number of positive SLNs. Mean absolute error and mean area under the ROC curve equals to 0.016 and 0.77 for the first, 0.023 and 0.75 for the second and 0.014 and 0.79 for the third nomogram. Three nomograms for predicting the likelihood of non-SLN metastases including the results of the preoperative US examination of the axilla were created at our institution. They differ in the inclusion of the results of intraoperative examination of SLNs and are thus applicable in different institutions. The
\end{abstract}

A. Perhavec · M. Hočevar · N. Besić · J. Žgajnar ( $₫)$ Department of Surgical Oncology, Institute of Oncology Ljubljana, Zaloška Cesta 2, 1000 Ljubljana, Slovenia e-mail: jzgajnar@onko-i.si

\section{P. Perme}

Faculty of Medicine, Institute of Biomedical Informatics, University of Ljubljana, Ljubljana, Slovenia validation results seem promising and omission of completion ALND might be considered in patients with the probability of having non-SLN metastases of $10 \%$ or less.

Keywords Breast cancer - Sentinel lymph node · Prediction of non-sentinel lymph node metastases . Nomogram

\section{Introduction}

Breast cancer surgery has gradually become more conservative, from radical mastectomy as the only surgical option in breast cancer patients in the late nineteenth and early twentieth century to breast conserving surgery and sentinel lymph node (SLN) biopsy, which are standards of care in selected patients nowadays.

SLN biopsy has been proven to accurately stage lymph nodes in breast cancer patients with less morbidity and better quality of life as compared with the standard axillary lymph node dissection (ALND) [1, 2]. In the case of a positive SLN, completion ALND (cALND) is routinely performed because of the possibility of non-SLN involvement. In such cases, cALND may offer additional prognostic and/or therapeutic benefit. However, in more than $50 \%$ of SLN-positive patients, the SLN has been shown to be the only lymph node affected by cancer [3]. Therefore, it would be important to identify patients who have no additional lymph nodes affected by cancer in order to avoid unnecessary ALND and its sequels.

In order to avoid unnecessary ALND, different tools for predicting the likelihood of non-SLN metastases in breast cancer patients with a positive SLN have been developed [4-10]. Most of them were created based on the data of the single institution with specific patient population and 
protocols (for example specialities in surgical procedures, intraoperative examination of SLNs, pathological assessment of SLNs, etc). Therefore, it is not surprising that they often perform poor when validated outside the institution they were developed $[11,12]$. The most widely validated is the prediction tool created by van Zee et al. from the Memorial Sloan Cattering Cancer Center (MSKCC) [10]. Because of interinstitutional differences in intraoperative examination of SLNs, they created two nomograms, one with and the other without frozen section information. As a result, one nomogram may be used in institutions where frozen section is performed and the other in those where it is not, which widens the applicability of their tool. However, MSKCC nomograms have an important weaknesses-they do not include the information on preoperative US examination of axillary lymph nodes, which is known to be an important predictor of non-SLN involvement when SLN is positive [12-14].

The aim of our study was to create and validate a tool for predicting the likelihood of non-SLN involvement that would include the information on the preoperative US examination of axillary lymph nodes.

\section{Patients and methods}

From January 2000 to January 2009, 2188 SLN biopsies in patients with invasive breast cancer were successfully performed at the Institute of Oncology Ljubljana. Of those, $1360(62 \%)$ were free of tumor cells, and in $143(7 \%)$, only isolated tumor cells (ITC) were found at the definite histology. Metastases were detected in $685(31 \%)$ cases. cALND was performed in 549 patients. Of those, 15 (3\%) have ITC, 133 (24\%) micrometastasis and 401 (73\%) macrometastasis in SLN. In 136 patients, cALND was not performed due to various reasons (comorbidities, inclusion in multicenter studies, patient preference).

In the present study, overall 534 patients were included. The inclusion criteria were as follows:

1. invasive breast cancer,

2. clinically negative axillary lymph nodes,

3. SLN biopsy successfully performed,

4. at least one metastasis (micro or macro) in SLN and

5. cALND performed

Fifteen patients who have ITC detected in SLN and who underwent cALND were excluded from the study, because cALND in patients with ITC-positive SLNs is no longer indicated as a routine procedure [15].

Clinical and pathological data of patients were prospectively collected. The data include age, tumor type, tumor size, tumor grade, location of the tumor, ER, PR and Her-2 status, lymphovascular invasion, number of removed
SLNs, number of positive and negative SLNs, number of all lymph nodes removed, number of all positive lymph nodes, number of non-SLNs affected by micrometastases and macrometastases, metastases size of SLNs, perinodal invasion of metastases in SLNs, results of the touch imprint cytology (TIC) and results of the US of the axilla.

Three models for predicting the likelihood of non-SLN metastases have been created and validated:

1. First model - the nomogram suitable for our institution and other institutions where intraoperative examination of SLNs is not routinely performed in patients with low risk for SLN involvement $(n=534)$ : the model consider the results of intraoperative examination of SLNs, where it was performed and the metastasis size where it was not performed.

2. Second model - the nomogram suitable for institutions where intraoperative examination of SLNs is not performed $(n=534)$ : the results of intraoperative examination of SLNs were not included in this analysis.

3. Third model- the nomogram suitable for institutions where intraoperative examination of SLNs is standardly performed $(n=460)$ : patients with SLNs, which were not examined by intraoperative examination, were excluded from this analysis.

The clinicopathological characteristics of patients are shown in Table 1.

Preoperative US examination of axillary lymph nodes

At the Institute of Oncology Ljubljana, preoperative US of axillary lymph nodes in combination with FNAB is a routine (but not obligatory) examination in clinically lymph node negative breast cancer patients.

The preoperative US examination of the axillary lymph nodes was performed by experienced radiologists, using a linear-array transducer with range $12-15 \mathrm{MHz}$ (Power Vision 8000 model SSA-390A; Toshiba, Otawara, Japan).

Lymph node was considered US suspicious if one of the following criteria were met:

- the longitudinal-transverse axis ratio of the lymph node $<1.2$,

- lymph node hilus not seen or

- the cortex thickness larger than $3 \mathrm{~mm}$

In all patients with US suspicious lymph nodes, US-guided FNAB with a 21-G needle was performed, and two smears were prepared. In the case of a positive cytologic result, patients proceeded to the immediate ALND, in the opposite case, the SLN biopsy was performed. The details of preoperative US examination of axillary lymph nodes are described elsewhere [16]. 
Table 1 Clinicopathological characteristics of patients

\begin{tabular}{|c|c|c|c|}
\hline & & First and second model & Third model \\
\hline \multicolumn{2}{|l|}{ No. of patients } & 534 & 460 \\
\hline \multicolumn{2}{|l|}{ Age } & Mean 55; median 54; range $25-87$ & Mean 54; median 53; range $25-87$ \\
\hline \multicolumn{2}{|l|}{ Tumor size (mm) } & Mean 20.0; median 18; range 3-80 & Mean 20.8; median 19 ; range $3-80$ \\
\hline \multicolumn{2}{|l|}{ No. of LNs removed per patient } & Mean 18.7; median 18; range 5-61 & Mean 18.7 ; median 18 ; range $5-39$ \\
\hline \multicolumn{2}{|l|}{ No. of positive LNs per patient } & Mean 2.8; median 1; range $1-29$ & Mean 2.8; median 2; range $1-29$ \\
\hline \multicolumn{2}{|l|}{ No. of SLNs removed per patient } & Mean $2.1 ;$ median 2 ; range $1-9$ & Mean $2.1 ;$ median 2 ; range $1-9$ \\
\hline \multicolumn{2}{|l|}{ No. of positive SLNs per patient } & Mean 1.3 ; median 1 ; range $1-5$ & Mean 1.4 ; median 1 ; range $1-5$ \\
\hline \multirow[t]{2}{*}{ SLN metastases size } & Macrometastases & $401 / 534(75 \%)$ & $351 / 460(76 \%)$ \\
\hline & Micrometastases & $133 / 534(25 \%)$ & $109 / 460(24 \%)$ \\
\hline \multirow[t]{2}{*}{ Proportion of positive SLNs per patient } & $>0 \%$ and $<100 \%$ & $257 / 534(48 \%)$ & $213 / 460(46 \%)$ \\
\hline & $100 \%$ & $277 / 534(52 \%)$ & $247 / 460(54 \%)$ \\
\hline \multirow[t]{2}{*}{ Non-SLNs metastases } & Non-SLNs positive & $180 / 534(34 \%)$ & $159 / 460(35 \%)$ \\
\hline & Non-SLNs macrometastases & $160 / 534(30 \%)$ & $143 / 460(31 \%)$ \\
\hline \multirow[t]{3}{*}{ Tumor type } & $\mathrm{IDC}^{\mathrm{a}}$ & $453 / 534(85 \%)$ & $393 / 460(85 \%)$ \\
\hline & $\mathrm{ILC}^{\mathrm{b}}$ & $67 / 534(13 \%)$ & $55 / 460(12 \%)$ \\
\hline & Other & $14 / 534(3 \%)$ & $12 / 460(3 \%)$ \\
\hline \multirow[t]{3}{*}{ Grade } & 1 & $96 / 534(18 \%)$ & $74 / 460(16 \%)$ \\
\hline & 2 & $258 / 534(48 \%)$ & $224 / 460(49 \%)$ \\
\hline & 3 & $180 / 534(34 \%)$ & $162 / 460(35 \%)$ \\
\hline \multicolumn{2}{|l|}{$\mathrm{LVI}^{\mathrm{c}}$} & $197 / 534(37 \%)$ & $180 / 460(39 \%)$ \\
\hline \multirow[t]{2}{*}{ Hormonal status } & $\mathrm{ER}+$ & $463 / 534(87 \%)$ & $398 / 460(87 \%)$ \\
\hline & $\mathrm{ER}-$ & 71/534 (13\%) & $62 / 460(13 \%)$ \\
\hline
\end{tabular}

${ }^{\text {a }}$ Invasive ductal carcinoma

b Invasive lobular carcinoma

c Lymphovascular invasion

\section{SLN biopsy}

On the morning of surgery, $30-60 \mathrm{MBq}$ of ${ }^{99 \mathrm{~m}} \mathrm{TC}$-labelled nanocolloid $\left(\right.$ Nanocol ${ }^{\circledR}$ ) in $0.2 \mathrm{ml}$ saline, divided in two doses, was injected peritumorally at two sites. Static and dynamic lymphoscintigraphy was then performed. Hot spots in the regional lymph node basins were marked on the skin. After the induction of general anesthesia, $1 \mathrm{ml}$ of blue dye (Blue Patente V; Laboratorie Guerbet, Aulnaysous-Bois, France) was injected peritumorally at the same two sites. SLN excision was guided by a hand-held gamma probe (Navigator GPS System, USSC, Watertown, Massachusetts, USA) and/or by the bluestained afferent lymphatic channels. The excised SLN was measured for ex vivo radioactivity. Additional hot nodes were removed until the background radioactivity was less than $10 \%$ to the hottest ex vivo SLN.

In the palpable breast cancers, excised SLNs were standardly examined by the TIC. The cytopathologist examined the imprints and diagnosed them as negative, suspicious or positive. In the case of a positive result, the surgeon performed immediate cALND. In the case of nonpalpable breast cancer, TIC was performed only if the SLN seemed clinically suspicious.

All slices of SLNs were formalin fixed and embedded in paraffin. The slides were examined with $H \& E$ staining. For all negative SLNs, serial sections were evaluated with $\mathrm{H} \& \mathrm{E}$ and immunohistochemistry (IHC)-stained levels at $250 \mu \mathrm{m}$. IHC staining was performed using commercially obtained monoclonal anticytokeratin antibody, clone MNF 116 (Dako, Glostrup, Denmark). All non-SLNs were sectioned transversely at 2-3 $\mathrm{mm}$ and entirely embedded. One section was examined with one H\&E staining per paraffin block.

The details of SLN procedure are described elsewhere [17].

Statistical analysis

A logistic model was fitted to the data to evaluate the predictive ability of the covariates. Nomograms were created to assist in future predictions, and the area under the ROC curve was calculated. The models were validated using bootstrap methods [18]. The optimism was described by estimating the mean absolute error and the bias-corrected value of the area under the ROC curve. Performance of the nomograms is illustrated using a bootstrap 
calibration curve. The $P$ values (two-sided) under 0.05 are considered statistically significant. The statistical analysis was performed at the Institute of Biomedical Informatics using statistical software $\mathrm{R}$ for Windows version 2.6.2.

\section{Results}

The intraoperative examination of SLNs was performed in 460 patients. The remaining patients $(n=74)$ had nonpalpable tumors; the SLNs in those patients (according to the policy at our institution) were thus not examined by intraoperative examination. Three models were calculated.

First model - the nomogram suitable for our institution and other institutions where intraoperative examination of SLNs is not routinely performed in patients with low risk for SLN involvement

534 patients were included in the calculation of the first model. In patients with SLNs, which were not examined by intraoperative examination (patients with non-palpable tumors), metastasis size was used in the calculation instead of the results of intraoperative examination of SLNs (74 patients).

The results of multivariate logistic regression are shown in Table 2. A nomogram created based on this multivariate model is shown in Fig. 1. The area under the ROC curve equals to 0.78 . The proportions of patients with non-SLNs metastases for various predicted probability groups were calculated, the smoothed line through them is given in Fig. 2. In particular, there were $80(15.0 \%)$ patients with less than $10 \%$ probability for non-SLNs involvement and 10 of them $(12.5 \%)$ actually had non-SLNs metastases (8 macrometastases and 2 micrometastases).

The model was validated using the bootstrap validation method with 500 repetitions. The bias-corrected smoothed curve of the actual versus predicted probabilities is given in Fig. 2. The mean absolute error equals to 0.016. The average area under the ROC was 0.77 .

Second model-the nomogram suitable for institutions where intraoperative examination of SLNs is not performed

534 patients were included in the calculation of the second model. The results of intraoperative examination of SLNs were not included in this analysis.

The results of multivariate logistic regression are shown in Table 3. A nomogram created based on this multivariate model is shown in Fig. 3. The area under the ROC curve equals to 0.76 . The proportions of patients with non-SLNs metastases for various predicted probability groups were calculated, the smoothed line through them is given in Fig. 4. In particular, there were $64(12.0 \%)$ patients with less than $10 \%$ probability for non-SLNs involvement and 6 of them (9.4\%) actually had non-SLNs metastases (4 macrometastases and 2 micrometastases).

Table 2 First model-results of multivariate logistic regression testing the association of each variable with the likelihood of non-SLN metastases

\begin{tabular}{|c|c|c|c|}
\hline & Coefficient & SE & $P$ value \\
\hline Intercept & -1.856 & 0.340 & $<0.001$ \\
\hline Tumor size & 0.408 & 0.106 & $<0.001$ \\
\hline TIC/FS/MetSize $=$ positive $(\text { vs. negative })^{\mathrm{a}}$ & 1.477 & 0.253 & $<0.001$ \\
\hline $\mathrm{TIC} / \mathrm{FS} / \mathrm{MetSize}=$ suspicious $(\text { vs. negative })^{\mathrm{b}}$ & 0.774 & 0.579 & 0.182 \\
\hline $\mathrm{TIC} / \mathrm{FS} / \mathrm{MetSize}=$ not performed/micro $\left(\right.$ vs. performed/negative) ${ }^{\mathrm{c}}$ & 0.332 & 0.616 & 0.590 \\
\hline TIC/FS/MetSize $=$ not performed/macro (vs. performed/negative) ${ }^{\mathrm{d}}$ & 1.093 & 0.392 & 0.005 \\
\hline US axilla $=$ normal (vs. not performed) & -0.963 & 0.227 & $<0.001$ \\
\hline US axilla $=$ suspicious (vs. not performed) & -1.004 & 0.342 & 0.003 \\
\hline $\mathrm{LVI}=$ present (vs. absent) & 0.488 & 0.214 & 0.023 \\
\hline No. of neg SLN $=1$ (vs. No. $=0$ ) & -0.347 & 0.234 & 0.139 \\
\hline No. of neg SLN $=>1($ vs. No. $=0)$ & -1.155 & 0.322 & $<0.001$ \\
\hline
\end{tabular}

Tumor size pathological tumor size in centimeters; TIC/FS/MetSize touch imprint cytology/frozen section/metastasis size in SLN; US axilla results of preoperative US examination of axillary lymph nodes, LVI lymphovascular invasion, No. of neg SLN number of negative SLN

${ }^{\text {a }}$ TIC or FS performed and positive (vs. performed and negative)

b TIC or FS performed and suspicious (vs. performed and negative)

c TIC or FS not performed, micrometastasis found in SLN (vs. TIC or FS performed and negative)

d TIC or FS not performed, macrometastasis found in SLN (vs. TIC or FS performed and negative) 


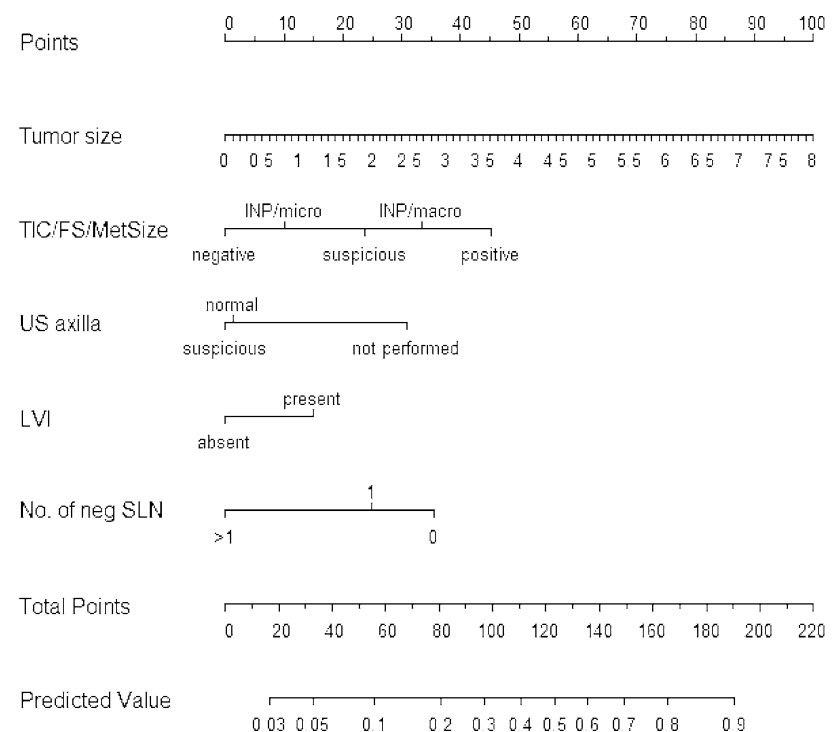

Fig. 1 The first nomogram - suitable for our institution and other institutions where intraoperative examination of SLNs is not routinely performed in patients with low risk for SLN involvement. Rows 2 through 6 represent variables. Vertical line should be made between each variable and the uppermost row (Points). In this way, the effect of each variable is determined by a defined number of points, which should be summed and located in row 7 (Total Points). Vertical line should be made between the row 7 and 8 (Predicted Value) to get the predicted probability of non-SLN metastasis. Tumor size-pathological tumor size in centimeters; TIC/FS/MetSize-results of touch imprint cytology (TIC) or frozen section (FS), when they were performed and the information on SLN metastasis size (MetSize), when TIC or FS were not performed: INP/micro-imprint (or FS) not performed, micrometastasis found in SLN; INP/macro-imprint (or FS) not performed, macrometastasis found in SLN; US axillaresults of preoperative US examination of axillary lymph nodes; LVI-lymphovascular invasion; No. of neg SLN-number of negative SLN: $>1$-more than 1 negative SLN, $1-$ one negative SLN, 0 - no negative SLN

The model was validated using the bootstrap validation method with 500 repetitions. The bias-corrected smoothed curve of the actual versus predicted probabilities is given in Fig. 4. The mean absolute error equals to 0.023 . The average area under the ROC was 0.75 .

Third model - the nomogram suitable for institutions where intraoperative examination of SLNs is standardly performed

460 patients were included in the calculation of the third model. Patients with SLNs, which were not examined by intraoperative examination, were excluded from this analysis.

The results of multivariate logistic regression are shown in Table 4. A nomogram created based on this multivariate model is shown in Fig. 5. The area under the ROC curve equals to 0.79 . The proportions of patients with non-SLNs metastases for various predicted probability groups were

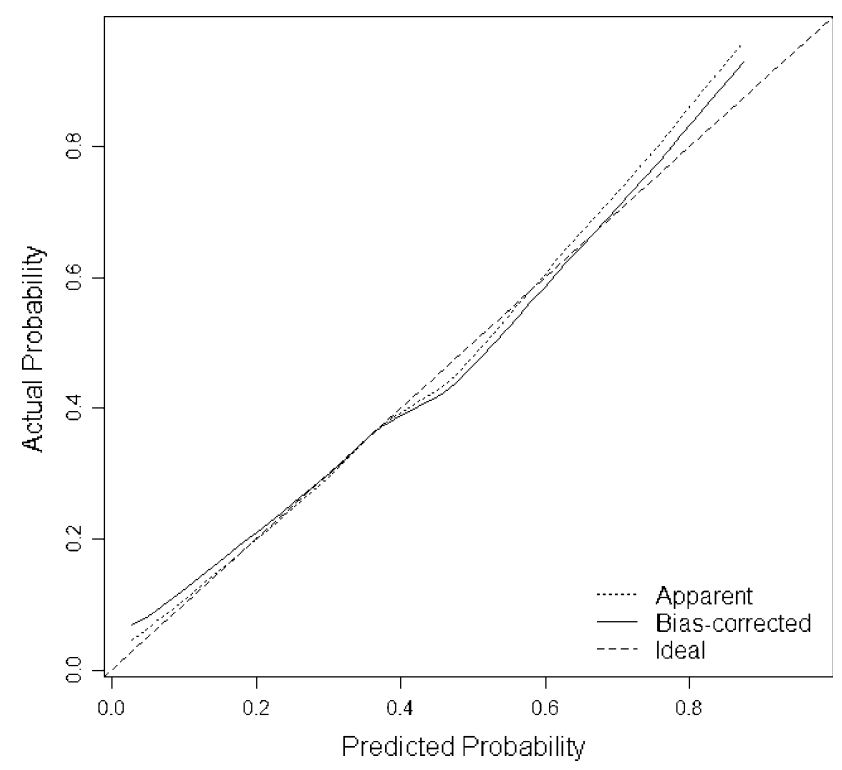

Fig. 2 Calibration plot for the first nomogram - the actual versus the predicted probabilites. The dashed line presents the line of equality, the dotted line depicts the observed situation on our sample and the solid line presents the bias-corrected line using the bootstrap validation procedure

Table 3 Second model-results of multivariate logistic regression testing the association of each variable with the likelihood of nonSLN metastases

\begin{tabular}{lrlr}
\hline & Coefficient & SE & $P$ value \\
\hline Intercept & -1.809 & 0.354 & $<0.001$ \\
Tumor size (cm) & 0.401 & 0.101 & $<0.001$ \\
MetSize (macro vs. micro) & 0.980 & 0.275 & $<0.001$ \\
US axilla = normal (vs. not performed) & -1.069 & 0.222 & $<0.001$ \\
US axilla = suspicious (vs. not & -1.012 & 0.334 & 0.003 \\
$\quad$ performed) & & & \\
LVI = present (vs. absent) & 0.549 & 0.209 & 0.009 \\
No. of neg SLN $=1$ (vs. No. $=0)$ & -0.379 & 0.229 & 0.098 \\
No. of neg SLN $=>1$ (vs. No. $=0)$ & -1.100 & 0.319 & $<0.001$ \\
No. of pos SLN $=>1$ (vs. No. $=1)$ & 0.451 & 0.220 & 0.041 \\
\hline
\end{tabular}

Tumor size pathological tumor size in centimeters, MetSize metastasis size in SLN: micro-micrometastasis, macro-macrometastasis; US axilla results of preoperative US examination of axillary lymph nodes, LVI lymphovascular invasion, No. of neg SLN number of negative SLN, No. of pos SLN number of positive SLN

calculated, the smoothed line through them is given in Fig. 6. In particular, there were $75(16.3 \%)$ patients with less than $10 \%$ probability for non-SLNs involvement and 8 of them (10.7\%) actually had non-SLNs metastases (6 macrometastases, 2 micrometastases).

The model was validated using the bootstrap validation method with 500 repetitions. The bias-corrected smoothed curve of the actual versus predicted probabilities is given in 


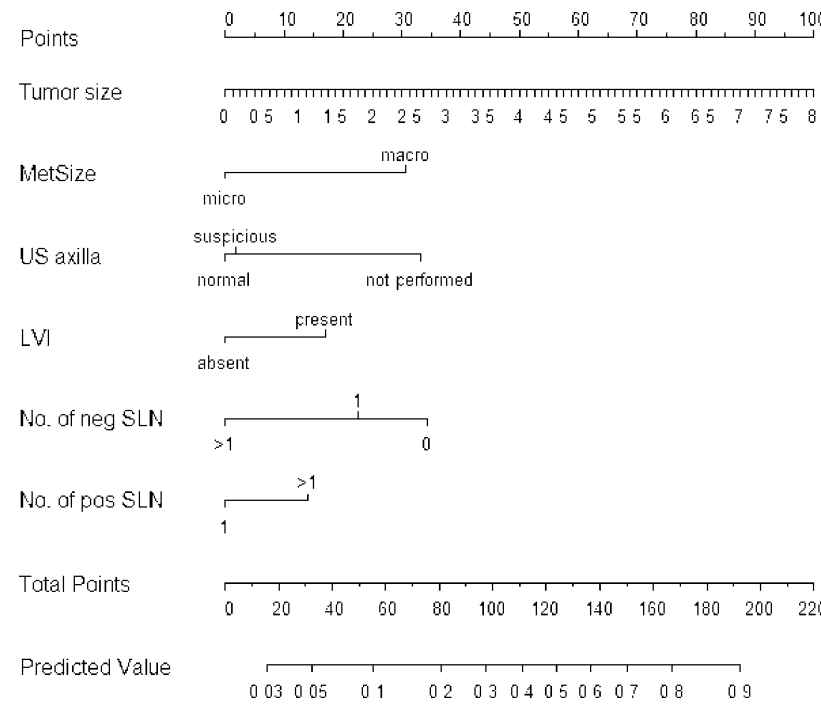

Fig. 3 The second nomogram-suitable for institutions where intraoperative examination of SLN is not performed. Rows 2 through 7 represent variables. Vertical line should be made between each variable and the uppermost row (Points). In this way, the effect of each variable is determined by a defined number of points, which should be summed and located in row 8 (Total Points). Vertical line should be made between the row 8 and 9 (Predicted Value) to get the predicted probability of non-SLN metastasis. Tumor size-pathological tumor size in centimeters; MetSize-metastasis size in SLN: micro-micrometastasis, macro-macrometastasis; US axillaresults of preoperative US examination of axillary lymph nodes; LVI-lymphovascular invasion; No. of neg SLN-number of negative SLN: $>1$-more than 1 negative SLN, 1 - one negative SLN, 0 no negative SLN; No. of pos SLN-number of positive SLN: 1 -one positive SLN, $>1$ more than one positive SLN

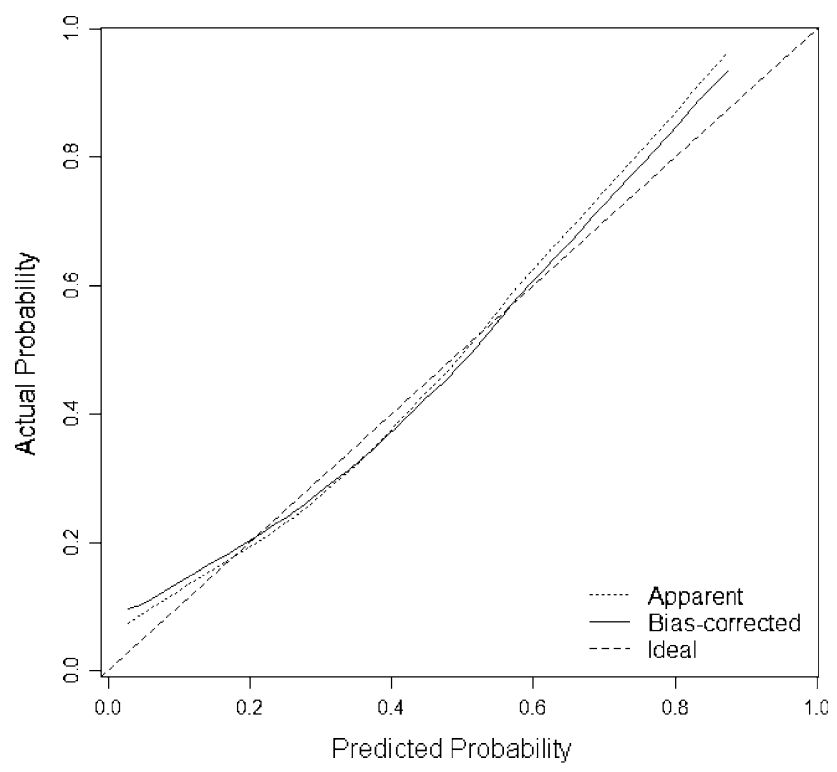

Fig. 4 Calibration plot for the second nomogram - the actual versus the predicted probabilites. The dashed line presents the line of equality, the dotted line depicts the observed situation on our sample and the solid line presents the bias-corrected line using the bootstrap validation procedure
Table 4 Third model-results of multivariate logistic regression testing the association of each variable with the likelihood of nonSLN metastases

\begin{tabular}{lrrr}
\hline & Coefficient & SE & $P$ value \\
\hline Intercept & -1.675 & 0.349 & $<0.001$ \\
Tumor size (cm) & 0.346 & 0.107 & 0.001 \\
TIC/FS = positive (vs. negative) & 1.415 & 0.257 & $<0.001$ \\
TIC/FS = suspicious (vs. negative) & 0.646 & 0.588 & 0.272 \\
US axilla = normal (vs. not performed) & -1.144 & 0.249 & $<0.001$ \\
US axilla = suspicious (vs. not & -1.170 & 0.359 & 0.001 \\
$\quad$ performed) & & & \\
LVI $=$ present (vs. absent) & 0.460 & 0.232 & 0.048 \\
No. of neg SLN = 1 (vs. No. $=0)$ & -0.444 & 0.256 & 0.083 \\
No. of neg SLN $=>1$ (vs. No. $=0)$ & -1.370 & 0.367 & $<0.001$ \\
No. of pos SLN $=>1$ (vs. No. $=1)$ & 0.477 & 0.245 & 0.052 \\
\hline
\end{tabular}

Tumor size pathological tumor size in centimeters; TIC/FS results of touch imprint cytology (TIC) or frozen section (FS), US axilla results of preoperative US examination of axillary lymph nodes, LVI lymphovascular invasion, No. of neg SLN number of negative SLN, No. of pos $S L N$ number of positive SLN

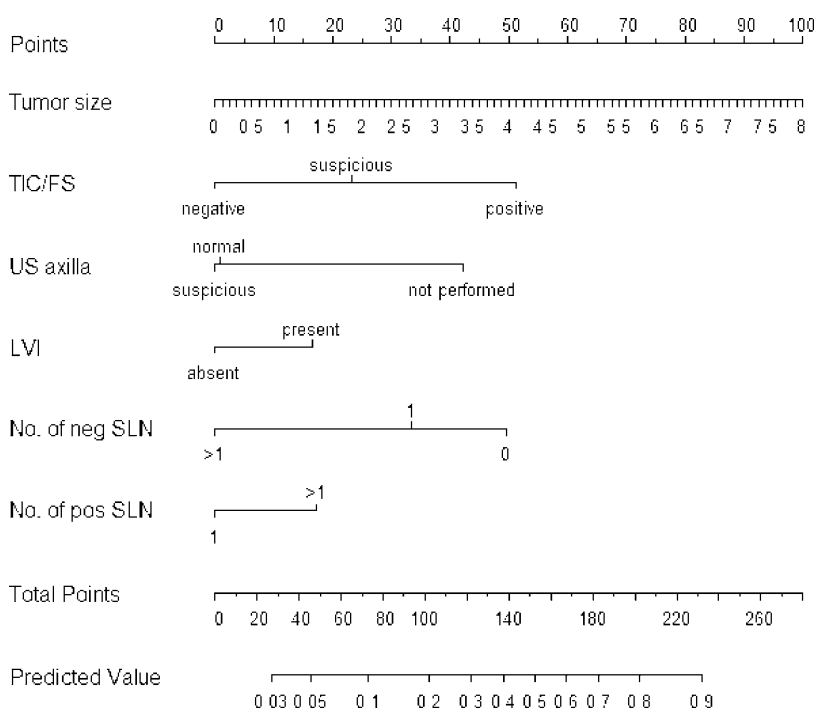

Fig. 5 The third nomogram-suitable for institutions where intraoperative examination of SLNs is standardly performed. Rows 2 through 7 represent variables. Vertical line should be made between each variable and the uppermost row (Points). In this way, the effect of each variable is determined by a defined number of points, which should be summed and located in row 8 (Total Points). Vertical line should be made between the row 8 and 9 (Predicted Value) to get the predicted probability of non-SLN metastasis. Tumor size-pathological tumor size in centimeters. TIC/FS-results of touch imprint cytology (TIC) or frozen section (FS); US axilla-results of preoperative US examination of axillary lymph nodes; LVIlymphovascular invasion; No. of neg SLN-number of negative SLN: $>1$-more than 1 negative SLN, 1 - one negative SLN, 0 -no negative SLN; No. of pos SLN-number of positive SLN: 1-one positive SLN, $>1$ more than one positive SLN 


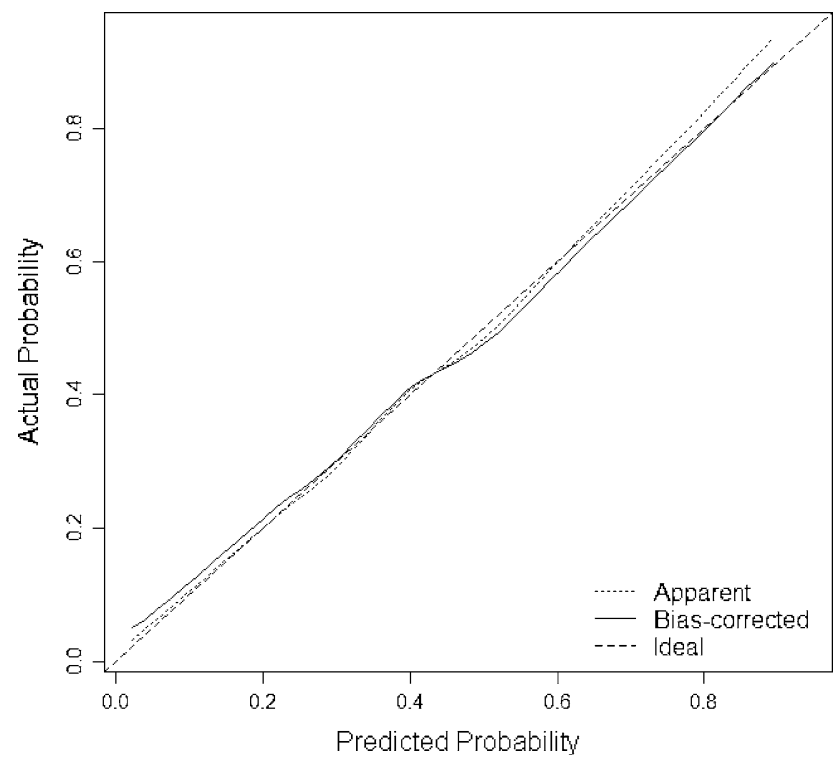

Fig. 6 Calibration plot for the third nomogram - the actual versus the predicted probabilites. The dashed line presents the line of equality, the dotted line depicts the observed situation on our sample and the solid line presents the bias-corrected line using the bootstrap validation procedure

Fig. 6. The mean absolute error equals to 0.014. The average area under the ROC was 0.79 .

36 patients have less than $10 \%$ probability of having non-SLN metastases according to all three nomograms. None of them have non-SLN macrometastases and 2 of them have micrometastases in one non-SLN.

\section{Discussion}

The SLN biopsy has been proven to reliably identify lymph node metastases in breast cancer patients [2]. About 50\% of patients with metastasis in SLN have additional metastases in non-SLNs [3]. Therefore, standard treatment in patients with metastatic SLN remains cALND or radiotherapy of the axilla [19]. However, not all patients with metastatic SLN have an equal possibility for non-SLN metastases. For example, is it necessary to further treat the axilla in patients with micrometastatic SLN, small tumors $(<1.5 \mathrm{~cm})$, more than two negative SLNs and preoperatively normal US examination of axillary lymph nodes? Furthermore, there is increasing number of older SLN-positive patients with many comorbidities or patients that are extremely unwilling to undergo further treatment of the axilla. A tool for predicting non-SLN metastases would be of great value in such cases for patients and clinicians.

Van Zee et al. from MSKCC created a nomogram for predicting non-SLN metastases in SLN-positive breast cancer patients. The authors concluded that the nomogram easily and accurately calculates the likelihood of having additional, non-SLN metastases for an individual patient [10]. However, many authors who validated the nomogram found several pitfalls, as for example, inclusion of the method of detection of SLN metastasis, which varies considerably among institutions. Furthermore, we found that the nomogram performed poorly when patients were grouped according to the information on the preoperative US examination of axillary lymph nodes. Namely, the nomogram overestimated the probability of non-SLN metastases in patients with preoperatively US uninvolved or US suspicious, but fine needle aspiration biopsy (FNAB) negative axillary lymph nodes. On the other hand, the nomogram underestimated the probability of non-SLN metastases in patients with preoperatively unperformed US of axillary lymph nodes [12]. This is not surprising, since US in combination with US-guided FNAB preoperatively detects metastases in axillary lymph nodes in $13 \%$ of SLN candidates at our institution [14]. The remaining patients with US negative axilla represent a distinct subgroup of patients with lower total axillary tumor burden and lower probability of non-SLN metastases when SLN is affected by cancer [14]. Based on these data, we created three new nomograms that also include the information on the preoperative US examination of axillary lymph nodes, which turned out as one of the most powerful variables. Surprisingly, the likelihood of non-SLN metastases is almost the same in patients with suspicious (but FNAB negative) as compared to those with normal US examination of axillary lymph nodes. However, patients with suspicious (but FNAB negative) US represent what is left of the entire US suspicious group after the patients with FNAB-positive result were already selected. Therefore, patients with suspicious (but FNAB negative) US have small tumor burden in axillary lymph nodes (ie, to small to be detected with US-guided FNAB), which explains our results.

The nomograms were created for different institutions, depending on the use of the intraoperative examination of SLNs. They have been developed based on the results of TIC as a method of intraoperative examination of SLNs. Thus, one might argue that our nomograms are not applicable for institutions, where frozen section is used instead of TIC. However, studies directly comparing frozen section with TIC showed that sensitivities of both methods are comparable [20, 21]. Furthermore, Lambert LA et al. who validated MSKCC nomogram (which uses frozen section), showed that TIC seems to be an acceptable substitute for frozen section as a nomogram variable [22]. We, therefore, believe that vice versa-substitution of frozen section for TIC-is also an acceptable option for our nomograms.

The first nomogram was created for our institution, where intraoperative examination of SLNs is performed only in selected patients; the second nomogram is suitable 
for institutions where intraoperative examination of SLNs is not performed and the third one where it is standardly performed. At our institution, intraoperative examination of SLNs is not routinely performed in patients in whom the positive result is less likely (patients with non-palpable tumors). As a result, the decision of performing or not performing the intraoperative examination of SLNs carries additional information. For this reason, we cannot simply use the second nomogram, when intraoperative examination of SLNs is not performed and the third one, when it is performed. Instead, the first nomogram should be used, which considers the information on intraoperative examination of SLNs, when it was performed and the information on SLN metastasis size, when it was not performed. Our opinion is that the first nomogram could also be used in other institutions with similar policy regarding the use of intraoperative examination of SLNs.

The SLN metastasis size, which is known to be strongly associated with non-SLN metastases [23-27], was statistically significant only when the results of intraoperative examination of SLNs were not available (the first model in patients in whom intraoperative examination of SLNs was not performed and the second model). It is known that TIC is less likely positive, when tumor burden in SLNs is small and is thus a surrogate of SLN metastasis size [17, 28]. In addition, patients with only clinically uninvolved axillary lymph nodes have larger tumor burden in SLNs than those with ultrasonically uninvolved axillary lymph nodes [14]. Information on US examination of axillary lymph nodes is thus another surrogate of SLN metastasis size. It is, therefore, not surprising that SLN metastasis size was not statistically significant, when both surrogates of SLN metastasis size were already included in the model.

The validation results of our nomograms as defined by the bootstrap corrected area under the ROC curve seem promising (first nomogram 0.77 , second nomogram 0.75 and third nomogram 0.79). The calibration curves showed that they are accurate through the entire range of probabilities, and the mean absolute calibration error is lower compared to other prediction tools [11]. We identified $12-16 \%$ of patients with the probability of having non-SLN metastases of less than $10 \%$ and found actual presence of non-SLN metastases in the expected range 10 (2 micrometastases)/80 (12.5\%), 6 (2 micrometastases)/64 (9.4\%) and 8 (2 micrometastases)/75 (10.7\%) for the first, second and the third model, respectively. Interestingly, none of them have macrometastatic disease in non-SLNs if the probability was less than $10 \%$ according to all three nomograms. Many of the low risk patients did not undergo cALND and were thus not included in the study. Therefore, the proportion of patients with the probability of having non-SLN metastases of less than $10 \%$ according to our nomogram is expected to be higher than calculated in this study.
Identifying those patients is important since most surgeons would omit the cALND when the probability of non-SLN metastases is below $10 \%$ [29]. Our opinion is that the risk of clinically evident axillary disease is very low in those patients. Namely, it is known that the false negative rate for SLN biopsy is between 5 and $10 \%$ [3, 30, 31]. However, the axillary recurrence rate in patients with a negative SLN biopsy and no further treatment of the axilla is far lower. A meta-analysis of 14 studies $(n=3802)$ reporting for axillary recurrence after negative SLN and no further treatment of the axilla showed a median axillary recurrence rate of $0.3 \%$. The median follow-up in those studies was 47 months [31]. Thus, considering SLN false negative rate of $5-10 \%$, only $3-6 \%$ of patients with residual disease in the axilla will have axillary recurrence after 47 months. If we decide not to further treat the axilla when the probability of non-SLN metastases according to the nomogram is $10 \%$ or less, about 100 out of 1,000 such patients will have residual disease in the axilla. Taking into account data on axillary recurrence after negative SLN, we can expect clinical manifestations in only 3-6 (0.3-0.6\%) of those patients. Furthermore, six series $(n=583)$ of selected SLN-positive patients who did not undergo cALND reported similar axillary recurrence $(0.5 \%$ at a median follow up of 31 months) [32-37]. These rates seem acceptably low and it is very unlikely that they would ever reach $10 \%$, the level at which local recurrence have a detectable impact on survival according to the latest Early Breast Cancer Trialists Collaborative Group overview [38]. Therefore, we believe that in patients with the possibility of having non-SLNs metastases of $10 \%$ or less omission of the cALND is a reasonable option. However, other factors such as patient's age and comorbidities, effectiveness of adjuvant therapy, delay in receiving adjuvant chemotherapy if cALND is performed and nonetheless patient's preferences should be considered besides the nomogram result and arbitrary defined cutoff value at which cALND might be omitted.

In conclusion, three nomograms for predicting the likelihood of non-SLN metastases, differing in the inclusion of the results of intraoperative examination of SLNs, were created at the Institute of Oncology Ljubljana. The nomograms include the information on the preoperative US examination of the axilla, which turned out as a powerful independent variable. The validation results for all three nomograms seem promising. Omission of cALND might be considered in patients with the probability of having non-SLN metastases of $10 \%$ or less, but further validations elsewhere are needed before the widespread use of the nomograms. Also, the nomogram results should be carefully discussed with the patient and other factors considered before final decision regarding further treatment of the axilla is made. 
Acknowledgments We are grateful to Matej Bračko, $\mathrm{PhD}$ and Snježana Frković-Grazio, PhD for acquiring some data.

\section{References}

1. Fleissig A, Fallowfield LJ, Langridge CI et al (2006) Post-operative arm morbidity and quality of life. Results of the ALMANAC randomised trial comparing sentinel node biopsy with standard axillary treatment in the management of patients with early breast cancer. Breast Cancer Res Treat 95:279-293

2. Veronesi U, Paganelli G, Viale G et al (2003) A randomized comparison of sentinel-node biopsy with routine axillary dissection in breast cancer. N Engl J Med 349:546-553

3. Kim T, Giuliano AE, Lyman GH (2006) Lymphatic mapping and sentinel lymph node biopsy in early-stage breast carcinoma: a metaanalysis. Cancer 106:4-16

4. Barranger E, Coutant C, Flahault A, Delpech Y, Darai E, Uzan S (2005) An axilla scoring system to predict non-sentinel lymph node status in breast cancer patients with sentinel lymph node involvement. Breast Cancer Res Treat 91:113-119

5. Degnim AC, Reynolds C, Pantvaidya G et al (2005) Nonsentinel node metastasis in breast cancer patients: assessment of an existing and a new predictive nomogram. Am J Surg 190:543550

6. Hwang RF, Krishnamurthy S, Hunt KK et al (2003) Clinicopathologic factors predicting involvement of nonsentinel axillary nodes in women with breast cancer. Ann Surg Oncol 10:248-254

7. Kohrt HE, Olshen RA, Bermas HR et al (2008) New models and online calculator for predicting non-sentinel lymph node status in sentinel lymph node positive breast cancer patients. BMC Cancer 8:66-80

8. Pal A, Provenzano E, Duffy SW, Pinder SE, Purushotham AD (2008) A model for predicting non-sentinel lymph node metastatic disease when the sentinel lymph node is positive. Br J Surg 95:302-309

9. Saidi RF, Dudrick PS, ReMine SG, Mittal VK (2004) Nonsentinel lymph node status after positive sentinel lymph node biopsy in early breast cancer. Am Surg 70:101-105

10. Van Zee KJ, Manasseh DM, Bevilacqua JL et al (2003) A nomogram for predicting the likelihood of additional nodal metastases in breast cancer patients with a positive sentinel node biopsy. Ann Surg Oncol 10:1140-1151

11. Coutant C, Olivier C, Lambaudie E et al (2009) Comparison of models to predict nonsentinel lymph node status in breast cancer patients with metastatic sentinel lymph nodes: a prospective multicenter study. J Clin Oncol 27:2800-2808

12. Zgajnar J, Perhavec A, Hocevar M et al (2007) Low performance of the MSKCC nomogram in preoperatively ultrasonically negative axillary lymph node in breast cancer patients. J Surg Oncol 96:547-553

13. Zgajnar J, Besic N, Podkrajsek M, Hertl K, Frkovic-Grazio S, Hocevar M (2005) Minimal risk of macrometastases in the nonsentinel axillary lymph nodes in breast cancer patients with micrometastatic sentinel lymph nodes and preoperatively ultrasonically uninvolved axillary lymph nodes. Eur J Cancer 41:244248

14. Zgajnar J, Hocevar M, Podkrajsek M et al (2006) Patients with preoperatively ultrasonically uninvolved axillary lymph nodes: a distinct subgroup of early breast cancer patients. Breast Cancer Res Treat 97:293-299

15. Samphao S, Eremin JM, El-Sheemy M, Eremin O (2008) Management of the axilla in women with breast cancer: current clinical practice and a new selective targeted approach. Ann Surg Oncol 15:1282-1296
16. Podkrajsek M, Music MM, Kadivec M et al (2005) Role of ultrasound in the preoperative staging of patients with breast cancer. Eur Radiol 15:1044-1050

17. Zgajnar J, Frkovic-Grazio S, Besic N et al (2004) Low sensitivity of the touch imprint cytology of the sentinel lymph node in breast cancer patients-results of a large series. J Surg Oncol 85:82-86

18. Harrell FE (2001) Regression modeling strategies. Springer, New York

19. Lyman GH, Giuliano AE, Somerfield MR et al (2005) American Society of Clinical Oncology guideline recommendations for sentinel lymph node biopsy in early-stage breast cancer. J Clin Oncol 23:7703-7720

20. Aihara T, Munakata S, Morino H, Takatsuka Y (2004) Comparison of frozen section and touch imprint cytology for evaluation of sentinel lymph node metastasis in breast cancer. Ann Surg Oncol 11:747-750

21. Brogi E, Torres-Matundan E, Tan LK, Cody HS III (2005) The results of frozen section, touch preparation, and cytological smear are comparable for intraoperative examination of sentinel lymph nodes: a study in 133 breast cancer patients. Ann Surg Oncol 12:173-180

22. Lambert LA, Ayers GD, Hwang RF et al (2006) Validation of a breast cancer nomogram for predicting nonsentinel lymph node metastases after a positive sentinel node biopsy. Ann Surg Oncol 13:310-320

23. Chu KU, Turner RR, Hansen NM, Brennan MB, Bilchik A, Giuliano AE (1999) Do all patients with sentinel node metastasis from breast carcinoma need complete axillary node dissection? Ann Surg 229:536-541

24. Kamath VJ, Giuliano R, Dauway EL et al (2001) Characteristics of the sentinel lymph node in breast cancer predict further involvement of higher-echelon nodes in the axilla: a study to evaluate the need for complete axillary lymph node dissection. Arch Surg 136:688-692

25. Reynolds C, Mick R, Donohue JH et al (1999) Sentinel lymph node biopsy with metastasis: can axillary dissection be avoided in some patients with breast cancer? J Clin Oncol 17:1720-1726

26. Viale G, Maiorano E, Mazzarol G et al (2001) Histologic detection and clinical implications of micrometastases in axillary sentinel lymph nodes for patients with breast carcinoma. Cancer 92:1378-1384

27. Weiser MR, Montgomery LL, Tan LK et al (2001) Lymphovascular invasion enhances the prediction of non-sentinel node metastases in breast cancer patients with positive sentinel nodes. Ann Surg Oncol 8:145-149

28. Perhavec A, Besic N, Hocevar M, Zgajnar J (2008) Touch imprint cytology of the sentinel lymph nodes might not be indicated in early breast cancer patients with ultrasonically uninvolved axillary lymph nodes. Ann Surg Oncol 15:2257-2262

29. Poirier E, Sideris L, Dube P, Drolet P, Meterissian SH (2008) Analysis of clinical applicability of the breast cancer nomogram for positive sentinel lymph node: the canadian experience. Ann Surg Oncol 15:2562-2567

30. Krag DN, Anderson SJ, Julian TB et al (2007) Technical outcomes of sentinel-lymph-node resection and conventional axillary-lymph-node dissection in patients with clinically nodenegative breast cancer: results from the NSABP B-32 randomised phase III trial. Lancet Oncol 8:881-888

31. Rutgers EJ (2008) Sentinel node biopsy: interpretation and management of patients with immunohistochemistry-positive sentinel nodes and those with micrometastases. J Clin Oncol 26:698-702

32. Fant JS, Grant MD, Knox SM et al (2003) Preliminary outcome analysis in patients with breast cancer and a positive sentinel lymph node who declined axillary dissection. Ann Surg Oncol $10: 126-130$ 
33. Guenther JM, Hansen NM, DiFronzo LA et al (2003) Axillary dissection is not required for all patients with breast cancer and positive sentinel nodes. Arch Surg 2003(138):52-56

34. Hwang RF, Gonzalez-Angulo AM, Yi M et al (2007) Low locoregional failure rates in selected breast cancer patients with tumor-positive sentinel lymph nodes who do not undergo completion axillary dissection. Cancer 110:723-730

35. Jeruss JS, Winchester DJ, Sener SF et al (2005) Axillary recurrence after sentinel node biopsy. Ann Surg Oncol 12:34-40

36. Langer I, Marti WR, Guller U et al (2005) Axillary recurrence rate in breast cancer patients with negative sentinel lymph node
(SLN) or SLN micrometastases: prospective analysis of 150 patients after SLN biopsy. Ann Surg 241:152-158

37. Naik AM, Fey J, Gemignani M et al (2004) The risk of axillary relapse after sentinel lymph node biopsy for breast cancer is comparable with that of axillary lymph node dissection: a followup study of 4008 procedures. Ann Surg 240:462-468

38. Clarke M, Collins R, Darby S et al (2005) Effects of radiotherapy and of differences in the extent of surgery for early breast cancer on local recurrence and 15-year survival: an overview of the randomised trials. Lancet 366:2087-2106 